PREHOSPITAL CARE

\title{
Retrospective observational case-control study comparing prehospital thrombolytic therapy for ST- elevation myocardial infarction with in-hospital thrombolytic therapy for patients from same area
}

\author{
M S V M Chittari, I Ahmad, B Chambers, F Knight, A Scriven, D Pitcher
}

Emerg Med J 2005;22:582-585. doi: 10.1136/emj.2004.020271

Objectives: To compare a system of prehospital thrombolytic therapy, delivered by paramedics under medical guidance, with in-hospital thrombolytic therapy in meeting National Service Framework (NSF) targets for treatment of acute myocardial infarction at a District General Hospital setting in England.

Design: Retrospective observational case-control study comparing patients with suspected acute myocardial infarction (AMI) treated with thrombolytic therapy in the prehospital environment with patients treated in hospital.

Setting: Wyre Forest District and Worcestershire Royal Hospital, UK.

Participants: (A) All patients who received prehospital thrombolytic therapy for suspected AMI accompanied by electrocardiographic features considered diagnostic.

(B) Patients who received thrombolytic therapy after arrival at hospital for the same indication, matched with group $A$ by age, gender and postcode.

Main outcome measures:

1. Call to needle time

2. Percentage of patients treated within one hour of calling for medical help

3. Appropriateness of thrombolytic therapy

4. Safety of thrombolytic therapy

Results: 1. The median call to needle time for patients treated before arriving in hospital $(n=27)$ was 40 minutes with an inter-quartile range 25-112 (mean 43 minutes). Patients from the same area who were treated in hospital $(n=27)$ had a median time of 106 minutes with an inter-quartile range 50-285 (mean 126 minutes). This represents a median time saved by prehospital treatment of 66 minutes.

2. 60 minutes after medical contact, $96 \%$ of patients treated before arrival in hospital had received thrombolytic therapy; this compares with $4 \%$ of patients from similar areas treated in hospital.

3. Myocardial infarction was confirmed in $92 \%(25 / 27)$ of patients who received prehospital thrombolytic therapy and similarly $92 \%$ (25/27) of those given in-hospital thrombolytic therapy.

4. No major bleeding occurred in either group. Group A suffered fewer in-hospital deaths than group B (1 versus 4). Cardiogenic shock (3 patients) and ventricular arrhythmia (5 patients) were seen only in group B.

Conclusion: Paramedic-delivered thrombolytic therapy can be delivered appropriately, safely, and effectively. Time gains are substantial and can meet the national targets for early thrombolytic therapy in the majority of patients.

\section{INTRODUCTION}

Early delivery of thrombolytic therapy after AMI saves lives and reduces morbidity. ${ }^{1-3}$ The NSF for coronary heart disease requires a standard "call to needle time" (from the initial call for help to treatment) of less than 60 minutes. In urban areas it may be possible to meet this target by rapid transfer and early administration of thrombolytic agents in hospital. In rural communities, however, where transfer times are often in excess of 30 minutes, the NSF document acknowledged that other models of care such as prehospital thrombolytic therapy might offer the best access to early treatment. ${ }^{4}$

In the Wyre Forest district, the closure of the coronary care facilities of a small district general hospital at Kidderminster - as part of the re-organisation of hospital services-led to introduction of prehospital thrombolytic therapy in May 2002 to meet the needs of a group of patients previously served by the hospital, and now exposed to longer journey times to the base hospital at Worcester. We describe the resulting system of prehospital thrombolytic therapy administered by paramedics under medical direction and compare it with the alternative option of thrombolytic therapy in hospital for a similar group of patients, with the NSF guidelines as standard.

\section{POPULATION AND METHODS \\ Background}

Front-line ambulances at the Kidderminster station that serves the Wyre Forest district were equipped with Mobimed systems to enable transmission of a 12-lead electrocardiogram (ECG), real-time ECG rhythm strip, heart rate, blood pressure and oxygen saturation measurements and transmit text messages to the base hospital. Paramedics based at this station received training in recording and transmission of ECGs, assessment of patients with suspected AMI and administration of thrombolytic therapy. Tenecteplase was chosen as the thrombolytic agent for this service because of the relative ease of administration as a single bolus, rather than infusion or double-bolus therapy. Prehospital thrombolytic therapy was considered if a patient with suspected AMI gave positive answers to all the 18 questions in the questionnaire developed by the Joint Royal Colleges Ambulance Liaison Committee (JRCALC). ${ }^{5}$ Advice to administer thrombolytic therapy (or not) was given by the on-call Medical Registrar or an experienced Senior House Officer

Abbreviations: $A M I$, acute myocardial infarction; $E C G$, electrocardiogram; JRCALC, the Joint Royal Colleges Ambulance Liaison Committee; NSF, National Service Framework; PCl percutaneous coronary intervention, ; STEMI, ST segment elevation myocardial infarction 
based at the base hospital. The decision was based on the details of the history, described by the paramedic in the text message, and on the presence or absence of ST segment elevation on the transmitted 12-lead ECG.

Hereford and Worcester Ambulance Service began using the Mobimed system to transmit clinical information and ECG recordings in October 2000 and from May 2002, paramedics based at Kidderminster Ambulance Station began to administer prehospital thrombolytic therapy.

\section{Patients}

Between May 2002 and October 2003, 27 patients received prehospital thrombolytic therapy from this service (Group A). Each of these patients was matched for age, gender, approximate date of presentation and postcode where he/ she lives with a patient who had thrombolytic therapy administered for the same indication after arrival in hospital (Group B).

Some of these patients had not received prehospital thrombolytic therapy because they were attended by ambulances whose staffs were not trained in the delivery of this treatment at the time. This included some non-paramedic crews, some Kidderminster crews before training was complete, and some crews from neighbouring ambulance stations. Patients in whom the decision to give thrombolytic therapy was delayed for clinical reasons were not used as controls, but this group included some patients who called for help more than 6 hours after the onset of chest pain and who would not have been eligible for prehospital thrombolytic therapy under JRCALC guidelines. The decision to administer thrombolytic therapy to the patients in group B was made either by the same medical staff as those advising the paramedics or by an experienced doctor in the Emergency Department.

\section{Study method}

Two Clinical Fellows in Cardiology (MV and IA) examined the ambulance report sheets and hospital medical records of all patients in both groups. The diagnosis of ST segment elevation myocardial infarction (STEMI) was considered confirmed when we identified ST segment elevation on the ECG and subsequent elevation of Troponin T (above 0.10).

\section{RESULTS}

\section{Appropriateness of therapy}

Group A: Of 27 patients who received prehospital thrombolytic treatment for STEMI, the diagnosis of AMI was subsequently confirmed in 25 patients by troponin $\mathrm{T}$ release. When we examined the initial 12-lead ECGs from the 2 patients who had no rise in troponin $\mathrm{T}$ they were not considered diagnostic of STEMI. We concluded that appropriate prehospital thrombolytic therapy was administered in $92 \%$ of this group.

Group B: Of 27 patients who had in-hospital thrombolytic therapy during the same period for STEMI, AMI was confirmed in 25 patients by release of troponin $\mathrm{T}$. Retrospectively, the initial 12-lead ECG from 1 patient was not considered diagnostic of STEMI. In this group 96\% of patients received thrombolytic therapy that was considered appropriate, based on ECG appearances and in 92\% appropriate therapy was confirmed by biochemical evidence of AMI.

\section{Times recorded}

These are summarised in Table 1 . We observed a clear difference in the completeness with which ambulance crews recorded time details. $100 \%$ of these details were recorded in patients who received prehospital therapy compared to only $67 \%$ in group B.
Table 1 Median times (in minutes) recorded in the two groups

\begin{tabular}{lll}
\hline Median (mean) times & Group A & Group B \\
\hline Pain to call time & $42(152)$ & $87(387)$ \\
Response time & $6(12)$ & $15(17)$ \\
Transport time & $65(65)$ & $49(49)$ \\
Call to needle time & $40(43)$ & $106(126)$ \\
Pain to needle time & $77(134)$ & $241(485)$ \\
\hline
\end{tabular}

Data are derived from times recorded in $100 \%$ in group $A$ and $67 \%$ of times in group $B$.

\section{Call to needle time}

This is the time from the patient's initial call for medical help to the start of delivery of thrombolytic therapy.

Group A: The median call to needle time was 40 minutes with an inter-quartile range of 25-112 minutes.

Group B: The median call to needle time was 106 minutes with an inter-quartile range of 50-285 minutes.

Administration of prehospital thrombolytic therapy therefore resulted in a median time saving of 66 minutes, over patients receiving treatment after arrival in-hospital.

At the NSF target maximum call to needle time of 60 minutes, 96\% (26/27) of patients treated before arriving in hospital (Group A) had received thrombolytic therapy. This compares with $4 \%(1 / 27)$ who received thrombolytic therapy in less than 60 minutes in the group treated in-hospital (Group B).

\section{Response time}

This is the time taken for qualified help to reach the patient. The NSF target is a maximum 8-minute response time for all Category A calls, which include calls for chest pain.

Group A: Arrival time was recorded in 27/27 (100\%) of patients in group A and in 17/27 (67\%) of patients in group B. In group A the response time was less than the NSF target of 8 minutes in 16/27 (59\%) patients, and in group B a response time of less than 8 minutes was achieved in 7/17 (41\%) of those with recorded times.

\section{Complications \\ In group A}

- AMI during the same admission: 2

- Death: 1 (same admission)

- Arrythmia: (paroxysmal atrial fibrillation): 1

\section{In group $B$}

- AMI during the same admission: 3

- Death: 4 (same admission)

- Arrythmia:

- Ventricular tachycardia: 3

- Ventricular fibrillation and cardiac arrest: 2

- Cardiogenic shock: 3

- Persistent ST segment elevation suggesting failed thrombolysis: 1

- Recurrent chest pain without further infarction: 2

Table 2 Type of infarct

\begin{tabular}{lll}
\hline Infarct Type & Group A & Group B \\
\hline Anterior & $37 \%$ & $37 \%$ \\
Inferior & $56 \%$ & $59 \%$ \\
None & $7 \%$ & $4 \%$ \\
\hline
\end{tabular}




\begin{tabular}{lll|}
\hline \multicolumn{2}{l}{ Table 3} & \multicolumn{2}{l|}{ Size of infarct } \\
\hline Infarct Size & Group A (Mean) & Group B (Mean) \\
\hline Troponin T Release & $2.45(4.07)$ & $3.15(6.94)$ \\
CK Release & $780(1127)$ & $1079.5(1827.8)$ \\
\hline
\end{tabular}

No major bleeding occurred in either group.

\section{DISCUSSION}

We believe that this study is the first that has used a casecontrol series to compare prehospital with in-hospital thrombolytic therapy. Although our study contains relatively small numbers and could not be expected to provide the strength of evidence of a larger prospective randomised controlled trial, the phased development of delivery of prehospital thrombolytic therapy in Wyre Forest has allowed us to compare the time of treatment administration and examine the subsequent outcomes in two matched groups of patients.

In our series prehospital thrombolytic therapy resulted in much earlier delivery of treatment with a median time saving of 66 minutes, compared to thrombolytic therapy given after arrival in hospital. The results of previous randomised trials of thrombolytic therapy show an inverse relationship between the reduction in mortality and the length of time from onset of symptoms to treatment, ${ }^{6}$ and this has driven various approaches to try delivering treatment as early as possible. One such measure is the setting of standards in the UK NSF for Coronary Heart Disease for the timely delivery of treatment, and our study has demonstrated the ability of prehospital thrombolytic therapy to meet those standards, endorsing the findings from other UK centres (7, 8, and 9). Early thrombolytic therapy in the prehospital setting has been shown to have benefits in terms of coronary patency, left ventricular function, morbidity, and mortality. ${ }^{10-20}$ In a meta-analysis, Morrison et al found that prehospital thrombolytic therapy reduced the relative risk of all-cause hospital mortality by $17 \%$. This corresponded to an absolute risk reduction of $2 \%$, which translates to one life saved for every 62 patients with AMI who received pre-hospital rather than in-hospital thrombolytic therapy. ${ }^{21}$

Our series was too small to demonstrate a statistically significant mortality benefit. We have seen a clear trend towards a better outcome in the group that received prehospital therapy, in keeping with these previously published findings. Whilst the type of infarct was similar between the groups, it was evident that the prehospital group had limited release of both cardiac markers, Troponin $\mathrm{T}$, and CK. This would partly be due to shorter pain to call times in this group, but also would reflect the early delivery of thrombolytic therapy. Whilst earlier delivery of thrombolytic therapy is likely to have contributed to this better outcome, there may have been other confounding factors. Every effort was made to ensure that in selecting matched patients who received in-hospital thrombolytic therapy, we chose those in whom clinical factors had not contributed to delay in delivery of treatment. However, approximately one third of patients receiving thrombolytic therapy after arriving in hospital had a recorded pain to call time of greater than 6 hours, so would not have met the JRCALC criteria for prehospital thrombolytic therapy. Also, we cannot completely discount the possibility that unrecorded clinical uncertainty about the relative risks and benefits of thrombolytic therapy may have been present and delayed the decision to give treatment in some patients in group B. Another possible confounding factor is that some patients treated in hospital received thrombolytic therapy with streptokinase rather than tenecteplase. Streptokinase may reopen the infarct-related artery in a slightly smaller percentage of patients than tenecteplase and as streptokinase is given as an infusion over one hour rather than a bolus injection, the completion of thromobolytic therapy is achieved later with this drug and this delay could also contribute to a less favourable outcome. It is therefore likely that the better outcomes seen in the group of patients who received prehospital thrombolytic therapy were due to a combination of interacting factors, of which earlier thrombolytic therapy was one of several.

However, this study has achieved its objectives. Not only does it demonstrate the ability of paramedics to achieve NSF targets for the prompt delivery of thrombolytic therapy, it also shows in our relatively small initial series that medicallydirected prehospital thrombolytic therapy was achievable, was at least as safe as thrombolytic therapy given after arrival in hospital and did not lead to more inappropriate treatment. This last conclusion is perhaps not surprising as the decision to give prehospital thrombolytic therapy was being made in most cases by the same doctors as those deciding on therapy after arrival in hospital. Following the success of the service in the Wyre Forest District, prehospital thrombolytic therapy has been extended to the rest of the counties of Herefordshire and Worcestershire served by the Ambulance Trust, and ongoing audit has shown further reductions in call-to needle times. The next step in developing prehospital thrombolytic therapy could be independent paramedic-led treatment and further evaluation will be needed to assess whether that can be achieved equally safely and effectively. A recent study from Devon ${ }^{22}$ showed that paramedics could record and interpret 12-lead ECGs and administer thrombolytic therapy safely in the community. In that study the calculated potential average time saved in delivery of thrombolytic therapy was 48 minutes per patient. The authors of that paper considered a physician-assisted model unreliable because of technological and communication failures due to poor mobile telephone signals, a problem that is not encountered in our service. Such transmission problems could be overcome by improvements in equipment and use of a more uniformly accessible communication system-for example, satellite-based transmission-but the move to a paramedic-led service would have the advantage of less reliance on technology and could potentially bring forward the delivery of treatment by a few more minutes by removing the time taken to transmit information and await a response. The longer transport times (mean difference 16 minutes) seen in our patients who received prehospital thrombolytic therapy probably reflect the time spent in assessment and delivery of thrombolytic therapy. In our local service the perceived advantage of the medically directed model was that it gave our paramedics the confidence to proceed swiftly to administration of thrombolytic therapy in all relevant patients, allowing the service to develop more quickly than it might have done if the paramedics had been expected to interpret ECGs themselves from the outset. From our experience we consider that the progression through medically directed to paramedic-led prehospital therapy is an effective method of introducing this service, whilst recognising that the direct introduction of a paramedic-led service may be necessary in some areas.

An interesting feature of our findings is that the time saved by prehospital thrombolytic therapy substantially exceeded the time taken to transport the patient to hospital. Shorter ambulance response times and more complete recording of response times were seen in the prehospital therapy group. The reason for this was not assessed, but may indicate that the training received by paramedics in prehospital treatment of AMI encouraged more rapid responses and more complete 
documentation. Other factors may have contributed. If some of the patients who received treatment in hospital were not attended by paramedic crews from their local station, it may be that their calls were received at a time when all available local paramedic crews were already busy and unable to respond with their usual speed. Nevertheless, a major advantage of the widespread introduction of prehospital therapy is that it removes any potential delays in the assessment and treatment of patients after arrival in hospital.

Delivery of prehospital thrombolytic therapy could be achieved widely throughout the UK. The major limits to its effectiveness would be either failure in some patients to achieve reopening of the culprit artery or later arterial reocclusion after successful initial thrombolysis. Primary percutaneous coronary intervention (PCI) can achieve a higher rate of arterial re-opening, can be performed safely and is being developed and promoted in some areas as the preferred approach to reperfusion for acute myocardial infarction. ${ }^{24}$ However, due to various geographic and resource constraints, this approach to treatment is not widely available yet.

Bennefoy et al compared the outcome from primary angioplasty with that following prehospital thrombolytic therapy and rescue angioplasty in the $25 \%$ of cases with suspected failed thrombolysis. ${ }^{25}$ This latter approach yielded the lowest mortality figures $(3.8 \%)$ recorded in the treatment of AMI in a large clinical trial, and suggested a $1 \%$ mortality advantage from early thrombolytic therapy and selected rescue angioplasty. The benefits of thrombolytic therapy and primary PCI may be complementary rather than mutually exclusive. Potential benefits of using thrombolytic therapy and/or platelet inhibitors before urgent facilitated PCI include rapid restoration of brisk coronary flow, enhanced tissue reperfusion, and greater myocardial salvage. It may be that optimal reduction in mortality and morbidity could be achieved by early paramedic-delivered thrombolytic and/or antithrombotic therapy and subsequent urgent PCI.

It is hoped that further studies will clarify the most effective early approach to therapy for patients with AMI, and that expansion of facilities for primary PCI will make this therapy available with appropriate speed to a much larger number of patients. For the present, early delivery of thrombolytic therapy remains the goal for attempting to achieve early reperfusion in the majority of patients. Our study adds to the body of evidence showing that paramedics in the prehospital setting can achieve this promptly, safely and effectively. We suggest that provision of prehospital thrombolytic therapy should be considered not only in rural communities, but also in urban areas, where traffic congestion may contribute to substantial delays in access to acute hospital services.

\section{Authors' affiliations}

M S V M Chittari, Worcestershire Royal Hospital, Worcester, UK I Ahmad, A Scriven, D Pitcher, Worcestershire Royal Hospital, Worcester UK

B Chambers, Director of Clinical Services, Hereford and Worcester Ambulance Service, Bransford, Worcester, UK

F Knight, Redditch Ambulance Station, Redditch,UK

Competing interests: none declared

Correspondence to: Dr Madhu Sudhan Varma Chittari Macharotu, Specialist Registrar Cardiology, West Midlands Rotation, University
Hospitals Coventry and Warwickshire NHS Trust, Coventry, CV2 2DX; madhuvarmacm@yahoo.co.uk

Accepted for publication 10 May 2005

\section{REFERENCES}

1 ISIS-2 (Second International Study of Infarct Survival) Collaborative Group. Randomised trial of intravenous streptokinase, oral aspirin, both, or neither among 17187 cases of suspected acute myocardial infarction: ISIS-2. Lancet 1988;2:349-360

2 Fibrinolytic Therapy Trialists' (FTT) Collaborative Group. Indications for fibrinolytic therapy in suspected acute myocardial infarction: collaborative overview of early mortality and major morbidity results from all randomised trials of more than 1000 patients. Lancet 1994;343:311-22.

3 GREAT Group. Feasibility, safety and efficacy of domiciliary thrombolysis by general practitioners: Grampian region early anistreplase trial: $B M J$ 1992;305:548-53.

4 Department of Health. National service framework for coronary heart disease. London: DoH; 2000, http://www.doh.gov.uk/coronary.htm. 5 Joint Royal Colleges Ambulance Liaison Committee/Ambulance Services Association. JRCALC, Thrombolysis update May 2004.

6 Boersma E, Maas ACP, Deckers JW, et al. Early thrombolytic treatment in acute myocardial infarction: reappraisal of the golden hour. Lancet 1996:348:771-75.

7 Kelly P. Thrombolysis in the pre-hospital setting. Br J Cardiol 2003;10:395-8.

8 Prendergast BD. Prehospital thrombolysis will pave the way for accelerated management of acute myocardial infarction. BMJ 2003;327:327-3.

9 Pedley DK, Bissett K, Connolly EM, et al. Prospective observational cohort study of time saved by pre hospital thrombolysis for ST elevation myocardial infarction delivered by Paramedics. BMJ 2003;327:22-6.

10 Weiss AT, Fine DG, Applebaum D, et al. Prehospital coronary thrombolysis: a new strategy in acute myocardial infarction. Chest 1987;92:124-128.

11 Bossaert LL, Demey HE, Colemont U, et al. Prehospital thrombolytic treatment of acute myocardial infarction with anisoylated plasminogen streptokinase activator complex. Crit Care Med 1988;16:823-830

12 Castaigne AD, Herve C, Duval-Moulin AM, et al. Prehospital use of APSAC: results of a placebo-controlled study. Am J Cardiol 1989:64:30A-33A.

13 Barbash GI, Roth A, Hod H, et al. Improved survival but not left ventricular function with early and prehospital treatment with tissue plasminogen activator in acute myocardial infarction. Am J Cardiol 1990;66:261-266.

14 Roth A, Barbash Gl, Hod H, et al. Should thrombolytic therapy be administered in the mobile intensive care unit in patients with evolving myocardial infarction? A pilot study. J Am Coll Cardiol 1990;15:932-936.

15 The Thrombolysis Early in Acute Heart Attack Trial Study Group. Very early thrombolytic therapy in suspected acute myocardial infarction. Am J Cardiol 1990;65:401-407

16 Weaver WD, Eisenberg MS, Martin JS, et al. Myocardial Infarction Triage and Intervention Project - phase I: patient characteristics and feasibility of prehospital initiation of thrombolytic therapy. J Am Coll Cardiol 1990; 15:925-931.

17 BEPS Collaborative Group. Prehospital thrombolysis in acute myocardial infarction: the Belgian eminase prehospital study (BEPS). Eur Heart $J$ 1991;12:965-967.

18 Applebaum D, Weiss AT, Koren G, et al. Feasibility of pre-hospital fibrinolytic therapy in acute myocardial infarction. Am J Emerg Med 1986;4:201-204.

19 European Myocardial Infarction Group (EMIP). Prehospital thrombolytic therapy in patients with suspected acute myocardial infarction. N Engl J Med 1993;329:383-9.

20 Koren G, Weiss AT, Hasin Y, et al. Prevention of myocardial damage in acute myocardial ischemia by early treatment with intravenous streptokinase. N Engl J Med 1985;313:1384-1389.

21 Morrison LJ, Verbeek PR, Mc Donald AC, et al. Mortality and pre hospital thrombolysis for acute myocardial infarction. A Meta- analysis. JAMA 2000;283:2686-92.

22 Keeling P, Hughes D, Price L, et al. Safety and feasibility of prehospital thrombolysis carried out by paramedics. BMJ 2003;327:27-28.

23 Ross AM, Coyne KS, Reiner JS, et al. A randomized trial comparing primary angioplasty with a strategy of short-acting thrombolysis and immediate planned rescue angioplasty in acute myocardial infarction: the PACT trial. PACT investigators. Plasminogen-activator Angioplasty Compatibility Trial. J Am Coll Cardiol 1999;34:1954-1962.

24 Grines CL, Browne KF, Marco J, et al. A comparison of immediate angioplasty with thrombolytic therapy for acute myocardial infarction. The Primary Angioplasty in Myocardial Infarction Study Group. N Engl J Med 1993;328:673-679.

25 Bonnefoy $\mathrm{E}$, Lapostolle $\mathrm{F}$, Leizorovicz A, et al. Primary angioplasty versus prehospital fibrinolysis in acute myocardial infarction: a randomised study. Lancet 2002;360:825-829. 